\title{
El Hombre y la Creación de Sentido: El camino de la Construcción de Significados en Schutz
}

\section{Man and the Creation of Sense: the way to Meaning Construction in Schutz}

\author{
Yasna Valenzuela Román ${ }^{1}$ \\ Universidad Santo Tomás de Chile
}

"Soy la única en saber de qué azul es el chal azul de esa mujer joven en este libro. Soy la única también que ve su sonrisa y su mirada. Sé que nunca podré describiroslo, hacéroslo ver. Nunca a nadie."

(Marguerite Duras, La vida material)

(Recepción: octubre 2004 - Aceptación: noviembre 2004)

\begin{abstract}
Este artículo trabaja la idea de significado desde la perspectiva de Schutz. Se pretende mostrar cómo se crea el sentido, y cómo interpretamos los significados de otros mediante esquemas de interpretación congruentes, es decir, cómo se construye lo intersubjetivo. En el primer punto se analizará la idea husserliana del movimiento de la razón latente a la razón develada, en tanto proceso de constitución de la subjetividad. En el segundo, se pretende mostrar la forma de objetivación de la intersubjetividad como un despliegue hermenéutico de la propia interpretación a la interpretación de los otros. Finalmente, se esboza una revisión crítica a la concepción de vida cotidiana planteada por la fenomenología, como punto de partida del quehacer de las ciencias sociales.
\end{abstract}

Palabras claves: Intersubjetividad, sentido, interpretación.

The article works the concept of meaning from the perspective of Schultz. The intention is to show how sense is created and how we interpret the meanings of others through congruent interpretation schemes. In other words, how intersubjectivity is constructed. The first part of the article develops the Husserlian idea of movement from latent to manifest reason, as a process of construction of subjectivity. The intention of the second part is to show objectivation of intersubjectivity as a hermeneutic unfolding of the personal interpretation towards the interpretation of others. Finally, a critical revision of the daily life conception developed by phenomenology, as a departure point for the task of social sciences, is outlined.

Key words: Intersubjectivity, sense, interpretation.

¿Cómo se crea sentido?, ¿cómo se recrea?, ¿cómo poder interpretar un significado de otro?, ¿cómo se construye lo intersubjetivo?, ¿cuál es su conexión con el mundo social?, ¿cómo pasar de lo individual, intransferible, a una dinámica colectiva? y, sobre todo, ¿con qué herramientas conceptuales contamos para poder adentrarnos en ese proceso?

\footnotetext{
Psicóloga, Licenciada en Psicología, Universidad de Chile. Magíster en Psicología, mención Social y de la Personalidad, Universidade Federal de Río de Janeiro. $\mathrm{PhD}$ en Filosofia, mención Epistemología de las Ciencias Sociales, Universidad de Chile. $\mathrm{PhD}$ (c) en Psicología, Universidad de Chile.
}

Más que pura exterioridad, es en un especial recorrido interior donde se logra responder para seguir preguntando. Cada uno de nosotros es una infinita pregunta, dirá Bachelard ${ }^{2}$. Es en el espacio de la imaginación donde alcanzamos la autorealización sostendrá Sartre ${ }^{3}$. Pero, ¿con qué categorías es posible develar ese proceso?, dicho de otra manera, ¿cuáles son los requisitos

2 BACHELARD, Gastón. "LA FORMACIÓN DEL ESPÍRITU CIENTÍFICO”. Editorial Anagrama. España, 1986. Pág. 68.

3 SARTRE, Jean Paul. "LA IMAGINACIÓN". Editorial Amorrortu. Buenos Aires, 1979. Pág. 136. 
de una ciencia que se enfrente a seguir los pasos de la construcción de la vida material?

Es en ese contexto donde adquiere su real dimensión el diagnóstico que hace Husserl a las ciencias europeas, puesto que se necesita que las ciencias humanas no se hayan reducido a lo fáctico, no renuncien a mostrar al ser humano el camino de construcción de sus significados, que se abran al mundo de la vida y desplieguen desde él un foco que permita iluminar, aunque sea parcialmente, ese proceso.

Esa es la opción con que se interpretará el argumento de Schutz al citar a Voegelin: "la sociedad humana no es meramente un hecho o un suceso del mundo externo que puede ser estudiado por un observador como un fenómeno natural. Aunque la exterioridad es uno de sus componentes importantes es un conjunto, un pequeño mundo iluminado de significados desde dentro por los seres humanos que continuamente lo crean y lo sustentan como modo y condición de su autorealización."”

También es posible llegar a dicha proposición justificándola desde dentro, es decir, persiguiendo las preguntas tras las que Schutz mismo iba, recorriendo con él ese misterioso proceso de la existencia humana. ${ }^{5}$

\section{La asignación de significado}

Husserl ve un movimiento que va desde la razón latente a la razón develada, cuya tarea es descubrir mediante la fenomenología nuestra vida verdadera. De este modo, afirma que lo que se convierte en fenómeno está ya en nosotros, pero escondido. Aún no vivimos según esa vida verdadera $\mathrm{y}$, por ello, no vivimos según la razón ${ }^{6}$. Este proceso conlleva dos reducciones: la primera al mundo-de-la-vida, la segunda, una reducción trascendental. Ésta comprende en cierto sentido a todas las demás. En ella debo siempre regresar a mí mismo, a mi propia sub-

4 SCHUTZ, Alfred. "THE PROBLEM OF SOCIAL REALITY". Collected Papers. Tomo I. Martinus Nijhoff Press. The Hague. 1967. Pág. 336.

5 Como afirma Eric Voegelin en la continuación de la cita. SCHUTZ, Alfred. "THE PROBLEM OF SOCIAL REALITY". Collected Papers. Tomo I. Martinus Nijhoff Press. The Hague. 1967. Pág. 336.

6 "El racionalismo iluminista era, y es ciertamente, una ingenuidad, pero renunciar al racionalismo iluminista no significa renunciar al racionalismo auténtico". HUSSERL, Edmund. "LA CRISIS DE LAS CIENCIAS EUROPEAS”. Editorial Siglo XX. España, 1976. Pág. 133. jetividad para orientarme siempre hacia mi vida que busca la verdad, hacia esa vida que, detrás de todas las máscaras yo, en cierto modo, vivo. Como la vida cotidiana se puede oscurecer, ofuscar, debo recurrir a una subjetividad que pone todo entre paréntesis. ${ }^{7}$ Así, subjetividad no es aceptación de un ser ya dado sino constitución activa de sentido y subjetividad formadora. Esto no implica negar el mundo como dado sino darle un significado al mundo.

De esa forma, la historia tiene un sentido desde esa vida precategorial. Ir desde un pasado rememorado en un presente intencional a un futuro anticipado también desde el presente como expectativa. En esto existe un movimiento constante que requiere de la permanente fundación y asignación de sentido para adentrarlo en el mundo-de-la-vida, en ese ideal de racionalidad, que no son otra cosa que correlatos de la vida intencional.

Por otra parte, yo y mundo son momentos de perderse y recobrarse en la vida, de presencia y de ausencia. Es el ser humano el que otorga sentido, pero en tanto es el yo originario descubierto "en la evidencia, en la presencia, el núcleo del significado de la verdad en el tiem-

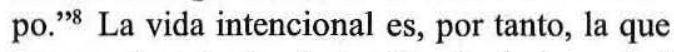
nos conduce hacia el significado de la verdad que da sentido a nosotros y al mundo.

Lo que en verdad vivenciamos en la duración, sostendrá Schutz, "no es un ser discreto y bien definido, sino una transición constante del ahora-así a un nuevo ahora-así." Es el proceso de reflexión el que nos permite clasificar esa vivencia dentro de conceptos de tiempo y espacio. Luego, podemos ver a los actos humanos desde este doble prisma, que siempre estará naciendo y muriendo. ${ }^{10}$ La captación de una vivencia en la corriente de la duración se cambia, en cada momento, al haberla recordado así. Luego, es este recuerdo el que aisla la vivencia de

7 Aquí se puede apreciar claramente la inserción fenomenológica en la estructura del tiempo.

8 PACI, Enzo. "FUNCIÓN DE LAS CIENCIAS Y SIGNIFICADO DEL HOMBRE". Editorial Fondo de Cultura Económica. México, 1984. Pág. 113.

9 SCHUTZ, Alfred. "FENOMENOLOGÍA DEL MUNDO SOCIAL”. Editorial Paidós. Buenos Aires, 1972. Pág. 75.

${ }^{10}$ Husserl denominará a este proceso, la doble intencionalidad de la corriente de la conciencia: intencionalidad transversal y longitudinal. SCHUTZ, Alfred. "FENOMENOLOGÍA DEL MUNDO SOCIAL”. Editorial Paidós. Buenos Aires, 1972. Pág. 76. 
la corriente de la duración y, modifica la captación, convirtiéndola en rememoración.

De ese modo, sólo en la evocación puedo tener repetido un objeto cultural idéntico. Como nos dirá Kundera: “ $¡ A$ Ah, qué daría yo porque pudieras ver el árbol!. Pero sólo yo lo tengo, aunque te lo mostrara no entenderías. Verías un tronco seco y gastado cuando para mí está fértil y es un retoño. Por eso es mejor separarnos. No sabes lo que puede ser un árbol."

Así, el acto de atención presupone una vivencia transcurrida, una vivencia que ya está en el pasado. Esta distinción es fundamental en la argumentación de Schutz. Se constituye en uno de los elementos claves de su crítica a Weber. Para él una de las limitaciones teóricas importantes en Weber será justamente no diferenciar adecuadamente el concepto acerca de qué es un acto significativo. ${ }^{12}$ Esta es la base de la distinción entre qué y cómo de una vivencia. Entre ese "estar ahí", en ese núcleo íntimo del yo que Scheler denomina "la privacía personal absoluta", necesariamente cerrada a toda convivencia posible y la evocación de un curso exterior de hechos. Esto implica, como expresara Neruda, ese ¡Al fin estamos juntos, yo con mi soledad y tú con la tuya!

Por otra parte, no toda vivencia es por naturaleza de asignación de significado. Se precisa que sean vivencias dadas en la intencionalidad, en forma de actividad espontánea o en una de sus modificaciones secundarias ya sea de retención o de reproducción. De esta forma, llega Schutz a definir la conducta "como una vivencia de la conciencia que confiere significados mediante actividad espontánea."13 Por ello, la vivencia fenoménica nunca es la conducta que uno tiene sino la conducta que uno ha tenido. La conducta consiste, entonces, en una serie de vivencias que se distinguen de las otras por una

12 "En esto resultan evidentes las limitaciones teóricas de Weber. Su concepto del acto significativo de un individuo, idea clave en la sociología comprensiva, de ninguna manera define un elemento primitivo, como él cree que lo hace. Es, por el contrario, una simple etiqueta para designar una zona muy compleja y ramificada que requiere más estudio."

SCHUTZ, Alfred. "FENOMENOLOGÍA DEL MUNDO SOCIAL”. Editorial Paidós. Buenos Aires, 1972. Pág. 37.

13 SCHUTZ, Alfred. "FENOMENOLOGÍA DEL MUNDO SOCIAL”. Editorial Paidós. Buenos Aires, 1972. Pág. 85. intencionalidad primordial de la "actividad espontánea que sigue siendo la misma en todas las modificaciones intencionales." 14

El significado de esas vivencias no es, por tanto, nada más que ese marco de interpretación que las ve como conducta. Ahora bien, la acción es una actividad espontánea orientada hacia el futuro. "En cada acción conocemos la meta por anticipado", sostendrá Husserl. ${ }^{15}$ Pero, tan pronto la mirada intencional ilumina la acción, la situación cambia. Entonces se contempla la acción como si ya hubiera transcurrido y terminado, totalmente constituida.

"Cuando uno escribe, hay como un instinto que juega. El escrito ya está en la noche. Escribir estaría en el exterior de sí, en una confusión de los tiempos: entre escribir y haber escrito, entre haber escrito y haber de escribir aún, entre saber e ignorar lo que es, partir del sentido pleno, sumergirse en él y llegar hasta el no-sentido. La imagen del cuaderno negro en medio del mundo no es aventurada. No es el paso del ser en potencia al ser en acto del que habla Aristóteles. No es una traducción. No se trata del paso de un estado a otro. Se trata del desciframiento de lo que ya es y ya ha sido gracias a uno, en el sueño de su vida. No es transferido, no se trata de esto. El instinto del que hablo consistiría en leer, ya antes de la escritura, lo que todavía es ilegible para los demás. Puedo decirlo de otro modo, puedo decir: sería leer su propia escritura, este primer estado del escrito de uno que aún es indescifrable para los demás. Condescender hacia la escritura de los demás para que el libro sea legible para ellos sería retroceder. Por mucho que se diga de otra manera y se empleen otras palabras, sería lo mismo. Delante de sí, uno tiene una masa entre vida y muerte que es de su dependencia. A menudo he tenido esa sensación de confrontación entre lo que ya estaba allí y lo que iba a estar en su lugar. Yo, en medio, arranco y transporto la masa que estaba allí. La rompo, es casi una cuestión muscular."16

${ }^{14}$ SCHUTZ, Alfred. "FENOMENOLOGÍA DEL MUNDO SOCIAL”. Editorial Paidós. Buenos Aires, 1972. Pág. 86.

is HUSSERL Edmund. "LÓGICA FORMAL Y LÓGICA TRASCENDENTAL”. Editorial UNAM. Máxico, 1962. Págs. 149 y ss.

${ }^{16}$ Duras, Marguerite. "LA VIDA MATERIAL". Editorial Plaza Janés. Barcelona, 1988. Págs. 31 y 32. 
Consecuentemente, todo proyecto de acción es más una fantasía de acción, una representación intuitiva. La distinción entre acción y conducta es que la acción es la ejecución de un acto proyectado. Luego, como sostiene Schutz, "el significado de cualquier acción es su correspondiente acto proyectado."17 Este es el sentido más específico de ese concepto formulado por Weber como "orientación para la acción." Luego, una acción carece de significado si se la separa del proyecto que la define. Por ello, " $¿$ de qué sirve hablar acerca del significado a que apunta una acción si ignoramos la fase de la acción que es importante para el actor y la sustituimos, a modo de interpretación, por un segmento arbitrariamente elegido del curso observado, que constituye "los hechos"? Así, para que un significado sea completamente significativo, requiere de un índice cronológico que especifique el momento de la interpretación.

Lo anterior implica que el significado es una elección. No en el sentido de necesidad sino de contingencia. Este proceso llevaría a configurar síntesis continuas y discontinuas. Este es uno de los puntos claves, a mi juicio, para hacer notar uno de los límites de Schutz. Él, siguiendo a Husserl, pretende explicar diferenciadamente el proceso de construcción de significados, pero en todo el proceso de significado como elección nunca contempla que existen otros factores que inciden directamente en esa elección como lo es el poder. Las significaciones, la manera en que consideramos la vivencia, en una sociedad moderna está traspasada por la ideología tecnológica y los mecanismos de información $^{18}$, que no son otra cosa que manifestaciones de poder. Pero éste no sólo puede ser identificado en un punto sino que constituye lo que Foucault denomina la microfísica del poder. ${ }^{19}$

17 SCHUTZ, Alfred. "FENOMENOLOGÍA DEL MUNDO SOCIAL". Editorial Paidós. Buenos Aires, 1972. Pág. 91.

18 En el sentido que Habermas le asigna a este proceso. Para un mayor análisis remito al texto de HABERMAS, Jürgen "CIENCIA Y TÉCNICA COMO IDEOLOGÍA". Editorial Tecnos. Madrid, 1984. Págs. 53 a 112.

19 "Entre cada punto del cuerpo social, entre un hombre y una mujer, en una familia, entre un maestro y un alumno, entre el que sabe y el que no sabe, pasan relaciones de poder que no son la proyección pura y simple del gran poder del soberano sobre los individuos; son más bien el suelo movedizo y concreto sobre el que ese poder se encardina. Para que el Estado funcione como funciona es necesario que haya del hombre a la mujer o del
Esta tensión existente al hablar de significado Schutz la ignora, adentrándose casi unilateralmente en las distinciones entre vivencia y significado.

Así, lo que el autor denomina "cambio atencional" puede estar sujeto a múltiples perspectivas. Lo que sí es básico es que los cambios se producen, pero es muy distinto que lo hagan por ruptura que por dejar traslucir un pentimento. ${ }^{20}$ Como fuere, la vivencia puede transformarse en un objeto dentro de la conciencia configurando un contexto de significado, pasando a formar parte de una síntesis de orden superior. Esto las diferencia de las configuraciones de orden inferior que son la simple atención a la vivencia y su duración. Es la configuración contextual la que hace que mis vivencias "sean mías."21 De este modo, "el significado a que apunta una vivencia no es nada más ni menos que una autointerpretación de esa vivencia desde el punto de vista de una nueva vivencia." ${ }^{22}$

Todo esto nos permite afirmar que el camino de construcción de una experiencia individual pasa por elaborar un contexto de significado, estructurarse sintéticamente y configurar el total de nuestra experiencia en el Aquí y Ahora. En este sentido, los esquemas de la experiencia son esquemas interpretativos. Ahora bien, existen dos clases de motivos: el motivopara que explica el acto en términos de proyecto y el motivo-porque que lo explica en función de las vivencias pasadas del actor. Es decir, los conocimientos, o la forma de conocer, se rela-

adulto al niño relaciones de dominación bien específicas que tienen su configuración propia y su relativa autonomía".

FOUCAULT, Michel. "LA MICROFÍSICA DEL PODER”. Editorial La Piqueta. Madrid, 1979. Pág. 104.

20 "La antigua pintura al óleo, al correr del tiempo, en ocasiones pasa a ser transparente. Cuando esto sucede, es posible, en algunos cuadros, ver los trazos originales: aparecerá un árbol a través del vestido de una mujer, un niño abre paso a un perro, un barco grande ya no se ve en un mar abierto. A esto se le llama "pentimento" porque el pintor se "arrepintió", cambió de idea. Quizá también sería correcto decir que la primitiva concepción, reemplazada por una preferencia posterior, es una manera de ver y luego ver una vez más".

HELLMAN, Lilian. "PENTIMENTO". Editorial Argos Vergara. Barcelona, 1978. Pág. 9.

21 SCHUTZ, Alfred. "FENOMENOLOGÍADELMUNDO SOCIAL”. Editorial Paidós. Buenos Aires, 1972. Pág. 104.

22 SCHUTZ, Alfred. "FENOMENOLOGÍADELMUNDO SOCIAL". Editorial Paidós. Buenos Aires, 1972. Pág. 107. 
ciona, de esa manera, con los intereses. ${ }^{23}$ Esta distinción no se efectúa en el caso weberiano por lo que da pie a una ambigüedad, al adoptar el concepto en un sentido laxo y enfatizar con ello sólo las interpretaciones ex post facto.

\section{La construcción de la intersubjetividad y el paso hacia el mundo social}

Esta experiencia que se abre puede contener en sí misma una objetivación en términos de una adecuación a normas externas por parte del sujeto. ${ }^{24}$ Entonces, si la experiencia individual es ya compleja, ¿cómo lograr conformar un contexto intersubjetivo? Husserl sostendrá que la individualidad verdadera requiere conquistarse dentro de una comunidad de individuos, es decir, descubrirse significa develar las relaciones que existen y nos constituyen. Por esa razón, la intersubjetividad deviene en tarea permanente. El significado siempre nos remitirá a la dialéctica del tiempo, la subjetividad y la intersubjetividad.

Por su parte, la intersubjetividad se despliega en cuanto abro mi interpretación a los significados de otros. En este proceso, es pertinente recordar que no es posible cosificar al otro dentro de un marco rígido de interpretación. Querer ser, es querer ser más sostendrá Nietzsche. Siempre mi interpretación del otro se hará desde mis vivencias, ordenando a la vez, mi propio contexto de significados, así como el otro ordenará el suyo. Se trata de un proceso de comprensión. Lo que puede captarse es siempre un valor aproximado del concepto límite: el significado al que otro apunta. De esta forma, podemos definir un signo como algo usado por una persona para expresar su vivencia. "Puesto que el signo se remonta a un acto de elección por parte de un ser racional, el signo es también una

23 Para un mayor análisis se remite a: HABERMAS, Jürgen. "CONOCIMIENTO E INTERÉS”. Editorial Taurus, Madrid, 1986.

${ }^{24}$ Como escribe Marguerite Duras: "Me pregunto en qué se basa la gente para contar su vida. Lo cierto es que hay tantos modelos de relatos que se hacen a partir del de la cronología, de los hechos exteriores. Se toma este modelo en general. Se parte del principio de la vida de uno y sobre las raíces de los acontecimientos, las guerras, los cambios de domicilio, las bodas, se desciende hacia el presente".

DURAS, Marguerite. "LA VIDA MATERIAL". Editorial Plaza Janés. Barcelona, 1988. Pág. 91. indicación de un hecho en la mente de quién usa el signo. A esto se le llama función expresiva del signo."25 Podemos concluir, por tanto, que los signos se interpretan no por esquemas externos sino de acuerdo a la experiencia pasada del intérprete y por esquemas pertenecientes a otros objetos.

Tanto la relación de comprensión de un sujeto a otro, como de un sujeto con objetos externos, tiene en común que solo existen como resultado de seres racionales. El proceso conceptual descrito nos contextualiza la noción de objetivo y subjetivo en Schutz.

El significado subjetivo significa que somos capaces de recapitular en nuestra mente los actos que constituyeron la vivencia del autor. El significado objetivo, en cambio, sólo podemos predicarlo del producto como tal, del contexto de significado ya constituido de la cosa producida. Visto así, el producto mismo no es un objeto sino el sedimento de una serie de hechos dentro de la mente del productor.

Como podemos apreciar, hasta ahora, todo el proceso descrito precisa de relaciones cara a cara, pero ¿cómo se produce la relación con ese mundo social complejo y multiforme? Un primer aspecto en el argumento de Schutz me parece crucial. Para poder acceder a ese nivel de configuración se requiere que el punto de partida de la ciencia social deba encontrarse en la vida social ordinaria. Es decir, expresa la idea husserliana entre la división existente entre sistema y mundo de la vida. Del límite de una ciencia que ha reducido la subjetividad a lo factual. Con ello se hace abstracción del sentido de la vida, separando el horizonte de la cientificidad de la vida humana. ${ }^{26}$ La sociología comprensiva, por tanto, debe partir del principio que "incluso a mis contemporáneos no puedo captarlos en sus vivencias sino inferir sobre la base de evidencia directa, las vivencias típicas que deben tener." ${ }^{27}$ Mediante este planteamiento se acerca Schutz al concepto de "tipo ideal weberiano."

25 SCHUTZ, Alfred. "FENOMENOLOGIA DEL MUNDO SOCIAL”. Editorial Paidós. Buenos Aires, Argentina, 1972. Pág. 149.

26 "La crisis de las ciencias se debe a la renuncia, por parte de las ciencias mismas, a la propia cientificidad, entendida como el horizonte de la vida, como sentido y propósito de la vida". HUSSERL Edmund. "LÓGICA FORMAL Y LÓGICA TRASCENDENTAL”. Editorial UNAM. México, 1962. Págs. 13.

27 SCHUTZ, Alfred. "FENOMENOLOGÍA DEL MUNDO SOCIAL”. Editorial Paidós. Buenos Aires, Argentina, 1972. Pág. 172. 
Pero el autor va más allá, enfatizando la prioridad de determinar cuáles son las esferas accesibles a los métodos de las ciencias sociales. Cuáles de ellos deben emplearse para hacer una investigación adecuada a sus objetos. Así por ejemplo, apartándose de Weber toma como punto de partida de la relación social, no la acción ni la conducta social, sino "las vivencias intencionales conscientes dirigidas hacia el yo del otro." ${ }^{28}$ Por eso, si al actuar sobre otro trato de que éste sepa que lo estoy haciendo, recién entonces existirá una relación de interacción. Una vez más, Schutz con esa argumentación nos retrotrae a la situación cara a cara como el único punto para deducir un mundo común e intersubjetivo. Esto conlleva el riesgo, como planteará Savater que el otro no pase a ser sencillamente "el lugar donde yo me reconozco."29 Para Schutz existen dos formas de comprender la conducta de otro en la manera típico-ideal, comenzando por el acto determinado, establecer el tipo de acción que lo produjo y, por último, determinar el tipo de persona que debe haber actuado de esa forma. $\mathrm{O}$, puedo invertir el proceso. Sin embargo, él mismo da cuenta de la dificultad de esta operación en términos de la variación de los tipos ideales y el desplazamiento del observador. Asimismo, alude al peligro de caer en antropomorfismos al personificar abstracciones tales como "estado", "la prensa", "la nación", "el pueblo".

A pesar de llegar a este punto, Schutz afirma que una relación social entre contemporáneos consiste en que cada uno de los partícipes aprehende al otro por medio de un tipo ideal y espera que el esquema de interpretación del otro sea congruente con el suyo. Lo que el autor no considera es que dentro de la sociedad moderna no sólo el sistema sino el mundo de la vida, se encuentra encapsulado, que las tradiciones vitales se han empobrecido y que, además, también son válidas para ellas el proceso de ruptura de las esferas valorativas y la pretensión de imposición, desde dentro, de la racionalidad instrumental. $^{30}$

${ }^{28}$ SCHUTZ, Alfred. "FENOMENOLOGÍA DEL MUNDO SOCIAL". Editorial Paidós. Buenos Ai res, 1972. Pág. 174.

${ }^{29}$ SAVATER, Fernando. "INVITACIÓN A LAÉTI CA". Editorial Anagrama. España, 1985. Pág. 68. ${ }^{30}$ HABERMAS, Jürgen. "CONCIENCIA MORAL Y ACCIÓN COMUNICATIVA". Editorial Penín sula. Barcelona, 1988. Pág. 75.
Así, la argumentación de Schutz encuentra otro límite ¿cómo poder generar, situándose solamente en el mundo de la vida, una noción de articulación que dé cuenta de la tensión existente entre diversidad y colectivo social? Pienso que éste es un esclarecimiento indispensable para perseguir el fin tras el que se encaminó la fenomenología, lograr que el acontecimiento se adentre más allá de las motivaciones tradicionales, que barra los pretextos heroicos o utilitarios que le servían de apoyo, para actuar o juzgar y que, por fin, "los rostros hayan devorado a las máscaras." "31

\section{Referencias}

BACHELARD, Gastón. “LA FORMACIÓN DEL ESPÍRITU CIENTÍFICO”. Editorial Anagrama. España, 1986. DURAS, Marguerite. "LA VIDA MATERIAL". Editorial Plaza Janés. Barcelona, 1988.

FOUCAULT, Michel. "LA MICROFÍSICA DEL PODER". Editorial La Piqueta. Madrid, 1979.

HABERMAS, Jürgen "CIENCIA Y TÉCNICA COMO IDEOLOGÍA". Editorial Tecnos. Madrid, 1984.

HABERMAS, Jürgen. "CONCIENCIA MORAL Y ACCIÓN COMUNICATIVA”. Editorial Península. Barcelona, 1988.

HABERMAS, Jürgen. "CONOCIMIENTO E INTERÉS". Editorial Taurus, Madrid, 1986.

HELLMAN, Lilian. "PENTIMENTO". Editorial Argos Vergara. Barcelona, 1978.

HUSSERL Edmund. "LÓGICA FORMAL Y LÓGICA TRASCENDENTAL”. Editorial UNAM. México, 1962. HUSSERL, Edmund. "LA CRISIS DE LAS CIENCIAS EUROPEAS”. Editorial Siglo XX. España, 1976.

PACI, Enzo. "FUNCIÓN DE LAS CIENCIAS Y SIGNIFICADO DEL HOMBRE". Editorial Fondo de Cultura Económica. México, 1984.

SARTRE, Jean Paul. "LA IMAGINACIÓN". Editorial Amorrortu. Buenos Aires, 1979.

SAVATER, Fernando. "INVITACIÓN A LA ÉTICA". Editorial Anagrama. España, 1985.

SCHUTZ, Alfred. "FENOMENOLOGÍA DEL MUNDO SOCIAL”. Editorial Paidós. Buenos Aires, 1972.

SCHUTZ, Alfred. "THE PROBLEM OF SOCIAL REALITY”. Collected Papers. Tomo I. Martinus Nijhoff Press. The Hague. 1967.

YOURCENAR, Marguerite. "ELECTRA O LA CAÍDADE LAS MÁSCARAS”. Editorial Lumen. Barcelona, 1986.
31 YOURCENAR, Marguerite. "ELECTRA O LA CAÍDA DE LAS MÁSCARAS". Editorial Lumen. Barcelona, 1986. Pág. 23. 